\title{
Controlling a Wheelchair Using Human-Computer Interaction
}

\author{
Ahmad Mahmoud ${ }^{1}$, Mohammad Hamoud ${ }^{2}$, Ahmad M. Ahmad ${ }^{3}$, Ahmad S. Ahmad ${ }^{4}$ \\ ${ }^{1,2,4} \mathrm{Al}$-Andalus University, Faculty of Medical Engineering, Al Qadmous - Tartus, Syria
}

${ }^{3}$ Al-Baath University, Department of Systems Engineering and Computer Networks, Homs, Syria

\begin{abstract}
In this paper, a prototype of an interactive wheelchair for quadriplegics and amputees is presented. Quadriplegics are people who are the victims of partial or complete paralysis of both upper and lower limbs; this is often the cause of a spinal cord injury or a disease in the area around the neck. The design presented in this paper is based on the field of Human-Computer Interaction, in which the face features of the quadriplegic are used to give him/her the opportunity to realize his/her needs. The face gestures are transformed to electrical signals using python programming language and the well-known OpenCv library for image processing. Signals are furthermore transmitted to an Arduino Uno microcontroller, which gives the orders to the wheelchair to move according to the patient's wish. The design was evaluated and the results were analysed.
\end{abstract}

Keywords: Human-Computer Interaction (HCI), Wheelchair Design, Arduino, OpenCv, Bluetooth.

\section{Introduction}

Disasters, crises and accidents make biomedical signal acquisition and processing a hot topic for researchers around the world. Despite the absence of statistics about the real number of quadriplegics and amputees, the number is increasing significantly. Quadriplegia results from the damage to the brain and/or the spinal cord that causes the loss of partial or total function of all four limbs. Researchers in this area aim to create trends and use means and technologies in order to fulfil patients' needs. One trend is to physically acquire bio-signals from muscles and transform them to motions; while another one is to express emotions and thoughts of the patient's brain as electric signals. The most attractive and simplest trend is to translate the gestures of the head or hands into electrical signals that represent the patient's wishs. This can be achieved based on the use of computers or microcomputers in addition to some specialized software, all of which forms what is known as HumanComputer Interaction (HCI). Human-computer interaction is a promising area of research nowadays. It is based on the design of systems and the use of computer technologies to perform some actions that correspond to the user's wishes. The user expresses his/her wishes through the interaction with these technologies [1], [2]. The interaction is based on the use of a Graphical User Interface (GUI), and the interaction at this stage is known as Loop of Interaction. The loop of interaction can be classified into two categories: an audio-based interaction and a visual-based interaction. Recognition of audio-based interaction is simpler to that of visual-based interaction, however, it is restricted because of the difference in accents among different users. Visual-based interaction is more attractive and is in common use when compared the other categories of HCI. Different methods to realize visual-based interaction can be used: hand gesture recognition, eye-gaze movement and head movement.

The wheelchair prototype presented here is based on a hybrid method. in fact, the wheelchair movement is connected with the movement of the head, where four regions of interest are defined. The main region is the entire face, two other regions are around the eyes and the last is around the lips. The computer webcam captures the face movement as a live video, which is then processed using OpenCv library. Next, the video is transformed into electrical signals, which, in turn, are transmitted wirelessly, using Bluetooth technology, to the microcontroller. The microcontroller receives these signals and transforms them into orders and commands to drive the wheelchair.

The rest of this paper is organized as follow: first, the literature review is presented; then, the proposed system is explained. The analysis and discussion are provided to conclude and present the future work.

\section{Related Work}

Many papers discussed the concept of Human-computer interaction for medical purposes. The authors in [3] investigated existing methods and presented a survey on head movement and eye-tracking detection systems. As the eye movement is a significant gesture, some authors took this movement as a basis for their design such as in [4], where the authors proposed a system that recognized eye movement from eye muscles. The proposed system generated signals, from different eye movements, directions and levels. Another way for designing such systems is to use brain signals, which express user's thought. The authors in [5] proposed a system that extracts signals from the brain using Electroencephalography (EEG). EEG is the basis for Brain Computer Interface (BCI), which extracts the user's brain signals that reflect his wishes to drive the machine. A mechanism for extracting signals from the brain was presented in [6]. The authors' objective was to design a brain-computer interface for non-vocal quadriplegics. They used an Emotiv EEG headset together with a piece of software. Using their system, the patient was able to do some actions on the computer. This was achieved controlling the 


\section{International Journal of Science and Research (IJSR) \\ ISSN (Online): 2319-7064}

Index Copernicus Value (2016): 79.57 | Impact Factor (2015): 6.391

mouse including the two mouse-click actions.

The authors in [7] developed a control system, based on the use of Atmega microcontroller, to move the wheelchair by using electrical signals generated by the user's face. Moreover, they developed an interface with an ocular keyboard, which enabled the patient to communicate with others by writing using eye movement.

\subsection{Bluetooth technology}

Bluetooth, or IEEE 802.15.1 [8], is a wireless technology used for data transfer over a short range at low power and low cost. It uses the radio frequency band between $2.4 \mathrm{GHz}$ and $2.485 \mathrm{GHz}$. It was initially invented by Ericsson in 1994 and was later standardized by the IEEE organization in 2009. The importance of Bluetooth is the ability to connect devices around the user and to form a Personal Area Network. 802. 15.1 can provide data transfer rates up to $4 \mathrm{Mbps}$ and has an ad-hoc nature; i.e. there is no need for an Access Point (AP). Bluetooth devices are first organized into a piconet of up to eight active devices. One of these devices is designated as the master, with the remaining devices acting as slaves. The slave can transmit only after the master has communicated with it. In addition to the slave devices, there can also be up to 255 parked devices. These devices cannot communicate until their status has been changed from parked to active by the master node.

\subsection{Arduino microcontroller}

Arduino is an open source computer hardware and software. It is considered to be of simple and low cost for designing embedded and digital systems. The Arduino project provides a simple IDE to easily program the microcontroller. At the heart of Arduino is an Atmel microcontroller. Arduino uses one or double-row female headers that facilitate the connection with another Arduino-based circuit called a shield. The most common shields are Wi Fi, Ethernet, motor and Bluetooth shields. For more insight into Arduino, please refer to [9].

\subsection{Open Source Computer Vision Library (OpenCV)}

OpenCV [10] is a real-time computer vision library developed by Intel in 1999. It is written in $\mathrm{C}++$ and is armed with a powerful API that simplifies the binding with MATLAB, Python and Java. OpenCV is used in a wide range of applications including: facial and gesture recognition system, HCI, robotics, object identification, etc. It is worth noting that OpenCV works with Windows, Linux and Mac OS and runs over different mobile Operating Systems such as Android and BlackBerry.

\section{The Proposed System}

This work is divided into two main parts: software development and hardware design. A high-level programming language, Python, is used because of its simplicity and because it is widely known for generalpurpose programming.
The architecture starts with acquiring face features using a laptop webcam and then processing the images on the laptop. The output from the image processing step is some electrical signals, which are transmitted wirelessly using Bluetooth technology to an Arduino Microcontroller. The controller, then, executes some commands on the wheelchair. Figure 1 depicts the block diagram of the design.

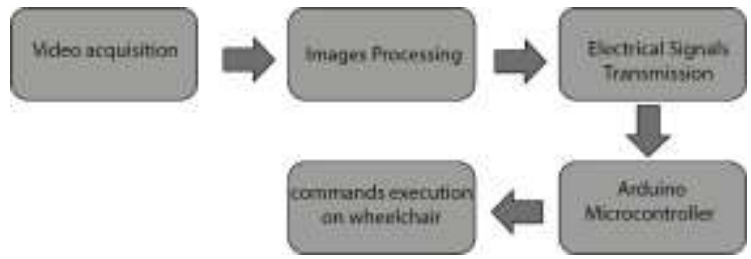

Figure 1: Designed System block diagram

\subsection{The Proposed Algorithm}

The proposed software design is composed of many modules:

\section{a. Application_arduino_bluetooth:}

This is a programming module that contains many functions and procedures responsible for communicating with the Bluetooth shield deposited over the Arduino Uno Microprocessor. The most important function is to discover the first Bluetooth device within a specific time given in seconds. Another function returns the name of all available Bluetooth devices and their addresses, and then presents this information as a dictionary. A class is provided to connect the laptop with one of the discovered devices, which is, in this case, the Bluetooth shield. This class, when the association with the Bluetooth device has been achieved, opens a socket from the laptop to the other side. This socket is used to send a message from the laptop and to receive it on Arduino via the Bluetooth connection. Another procedure is used to send the following commands: Forward, Backward, Right and Left to Arduino.

\section{b. application_commands}

In certain circumstances, some control commands could be wrong, or that certain level of noise is present. We define two classes to overcome this problem. In fact, we defined a class that represents a buffer with a fixed size, where the currently coming command replaces the old one. The most repeated command is considered to be the correct one if it surpasses a defined threshold (maxLength) and, as a consequence, this command is either sent to Arduino microcontroller, or not. This is used to reduce the probability of incorrect commands since some commands may have errors or noise while processing the images.

It is worth noting that a procedure is used to translate the commands into textual format printed on the laptop screen.

\section{c. application configuration}

We defined a class that contains constants used in different places within the program. These constants determine the locations of parameter files of algorithms that define faces, eyes and smiles. Some of these constants define the minimum dimensions of rectangles that specify the face, eye and smile that can be recognised within an image. Some other constants are used to define the colour degrees that form the basis for

\section{Volume 7 Issue 1, January 2018}




\section{International Journal of Science and Research (IJSR) \\ ISSN (Online): 2319-7064}

Index Copernicus Value (2016): 79.57 | Impact Factor (2015): 6.391

the rectangles that frame the detected face, eye and smile. As a matter of fact, all project configurations are grouped here in one place.

\section{d. application_roi}

It is very important to define the Region Of Interest (ROI). The ROI is a fixed rectangle within the image into which the user must fit the picture of his face. Many smaller rectangles appear within this ROI only when the face, eyes and smile are detected. This is achieved by defining $2 \mathrm{D}$ coordinates $\mathrm{X}$ and $\mathrm{Y}$. The decision is made after analysing the relationship between these small rectangles in relation of the two coordinates.

One of the main challenges encountered was to decide the true positions of the eyes and smile. This is achieved by defining a procedure that decides the true smile. A true smile is detected when the eyes are situated on a horizontal line, the right eye is on the right of the left eye and the smiling mouth is directly under the eyes in the centre. Once a true smile is detected, a command must be formed, which is achieved by defining another procedure that makes a decision in relation of the position of the eyes and smile. In other words, the position of the eyes, left or right, and the distance of the webcam is the deciding factor concerning the command.

We can summarize this procedure as follows:

- Take one frame, from the video stream, defined by file path or web camera.

- Crop the image and extract from it the area determined by RoiBound rectangle.

- Check if the eyes and smile are properly aligned.

- Comparing one object (eye) with another to check if they are at the same horizontal level.

- Decide which is the right eye and which is the left eye.

- Process RoiBounds objects, i.e. the position of each rectangle and deciding what the command (CMD) is based on the locations of objects.

\section{e. application_detect_main}

This is the main program that links all the previous modules and procedures.

We can summarize the overall algorithm as follows:

Image processing phase:

1) Receiving the image from the webcam.

2) Transforming the image so that the vertical symmetry is achieved. This means that the user sees the image on the laptop screen as if he was looking at a mirror.

3) Converting the coloured image into a 1-bit image because it can be processed quicker than the coloured (24-bit or 8bit) image under OpenCV.

4) Calling the algorithm of face detection / face annotation on the image currently being processed.

5) Calling the algorithm for determining the eyes and smile on the image.

\section{Command extraction}

1) Receiving the processed image from the image processing step.
2) Verifying the correctness of the image, i.e. the eyes are on the same horizontal line and the smile is under the eyes.

3) Determining which command is to be executed, depending on the position of the eyes and smile, in relation to the central point.

\section{Sending Command wirelessly and displaying:}

1. Sending the command using the procedure that achieves the connection with the Bluetooth shield on one hand, and the image is displayed on the laptop screen with rectangles around the eyes, smile and face on the other hand. Furthermore, a text is displayed on the screen showing the command being executed. There is an array of on/off circles on the top left area of the image, which can be used to help the patient.

It is worth noting that a "cascade" classifier is used in order to return the RoiBounds that represents the area of the detected object.

\subsection{Hardware components}

The hardware design is composed of the following:

- Arduino Uno microcontroller

- Bluetooth Shield

- Battery (9 V)

- Motor driver (L293D)

- 2 DC motors

- 2 wheels and 2 small gearboxes.

2 DC motors are used in the design for the sake of simplicity; however, stepper motors can be used. Figure 2 depicts the circuit diagram of the design.

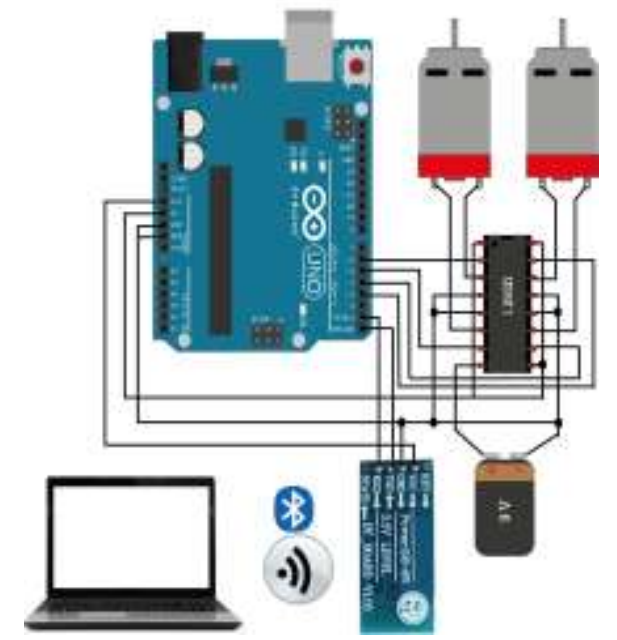

Figure 2: Circuit diagram

Figure 3 and Figure 4 depict the hardware design and the facial procedure for controlling the wheelchair respectively. 


\section{International Journal of Science and Research (IJSR) \\ ISSN (Online): 2319-7064}

Index Copernicus Value (2016): 79.57 | Impact Factor (2015): 6.391

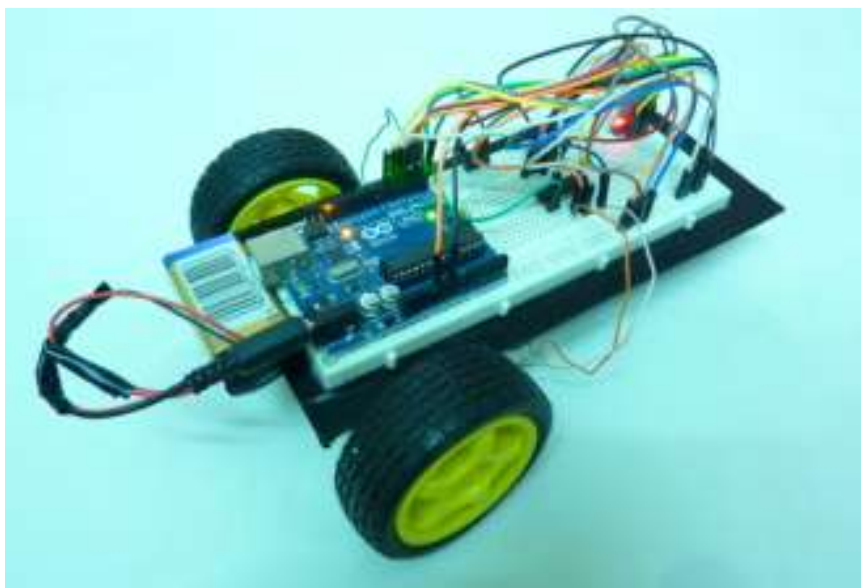

Figure 3: Proposed prototype

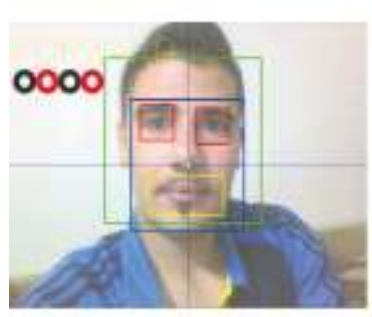

(a)

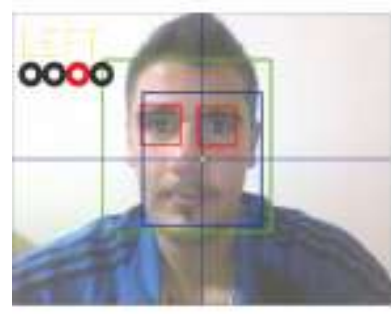

(b)

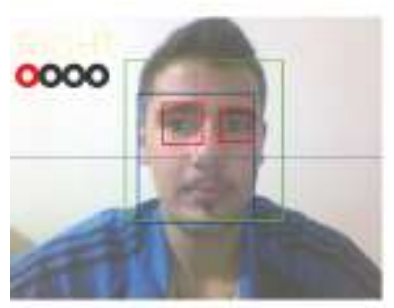

(c)

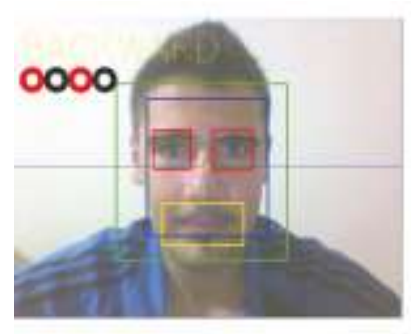

(d)
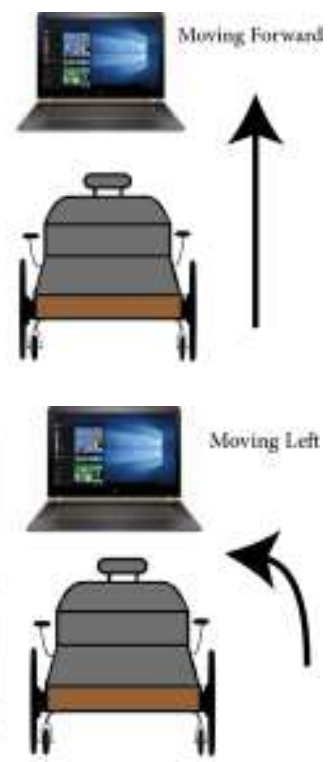

$($ )
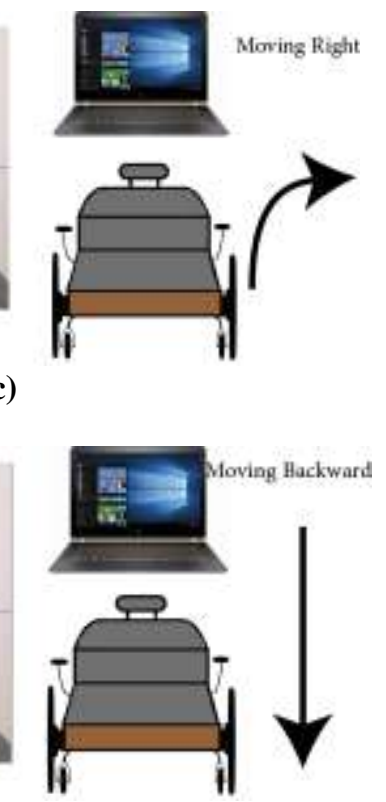

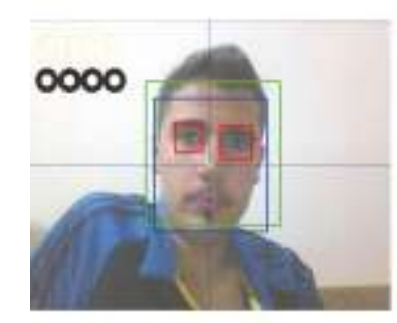

(e)

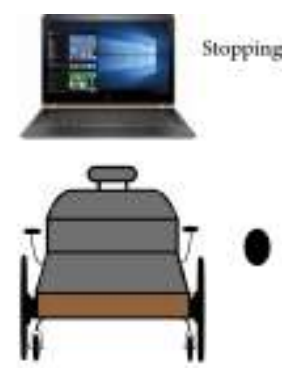

Figure 4: a) Moving Forward, b) Moving Left, c) Moving Right, d) Moving Backward and e) Stopping

\section{Analysis and Discussion}

The most motivating factor of this work was the need to define a "presentation service" that can solve the language problem. Relying on speech for such systems is not a practical solution because of the problem of interpreting different languages. Moreover, patients coming from different regions impose the problem of different accents. The idea of this design relies on translating the thoughts of patients into a commonly understood and person-independent language. This presentation service can be expressed by the procedure of reading the face gestures and transforming them into orders. This presentation service transforms the gestures into a common language that the software can understand. To sum up, relying on physical actions makes the product more practical and gives it a universal context.

One of the challenging issues was designing a user-friendly interface that can be accepted by different age groups on one hand, and evaluating the design to check whether this is what users really need on the other hand. So, the big question was: does the final product work as expected?

To answer this question, many tests were conducted. The tests conducted were about "usability". Usability refers to the effectiveness and the efficiency of the product on one hand, and the satisfaction of the user in a specified context on the other hand [11].

The evaluation process was divided into three phases. The first was related to the instantaneous interaction between the user and the system. While the second phase related to the psychological behaviour of the user, which was achieved by filming users while using the system. The final phase was conducting a satisfaction. It should be noted that the tests were conducted in an indoor environment; however, they can also be conducted in an outdoor environment.

In the first phase, we followed the same methodology reported in [12]. However, how many users are required to conduct the test? Depending on the professional recommendation stating that 5-12 testers are enough for such types of tests [11], both non-trained and trained (15-min training) users tested the design. The testing users were divided into three age groups: 7-15, 16-35 and 36-60 years, with 10 users in each group. The evaluation process included all the commands: Forward, Left, Right, Backward and Stop. The results are presented in Table 1. 


\section{International Journal of Science and Research (IJSR) \\ ISSN (Online): 2319-7064}

Index Copernicus Value (2016): 79.57 | Impact Factor (2015): 6.391

Table 1: Test results for both non-trained trained users: a) for (7-15)-year age group, b) for (16-35)-year age group and c) for (36-60)-year age group.

(a)

\begin{tabular}{|c|c|c|}
\hline Commands & Non-Trained Users (7-15) & Trained Users(7-15) \\
\hline Forward & $96 \%$ & $100 \%$ \\
\hline Left & $95 \%$ & $100 \%$ \\
\hline Right & $95 \%$ & $100 \%$ \\
\hline Stop & $93 \%$ & $100 \%$ \\
\hline Backward & $93 \%$ & $99 \%$ \\
\hline
\end{tabular}

(b)

\begin{tabular}{|c|c|c|}
\hline Commands & Non-Trained Users (16-35) & Trained Users (16-35) \\
\hline Forward & $93 \%$ & $100 \%$ \\
\hline Left & $91 \%$ & $100 \%$ \\
\hline Right & $93 \%$ & $99 \%$ \\
\hline Stop & $89 \%$ & $98 \%$ \\
\hline Backward & $86 \%$ & $96 \%$ \\
\hline
\end{tabular}

(c)

\begin{tabular}{|c|c|c|}
\hline Commands & Non-Trained Users (36-60) & Trained Users (36-60) \\
\hline Forward & $90 \%$ & $100 \%$ \\
\hline Left & $88 \%$ & $98 \%$ \\
\hline Right & $99 \%$ & $96 \%$ \\
\hline Stop & $84 \%$ & $96 \%$ \\
\hline Backward & $82 \%$ & $95 \%$ \\
\hline
\end{tabular}

It can be noted from Table 1 that the first age group is the quickest to understand and deal with the interface using face gestures. The table also shows, as one would expect, that the third age group is the slowest, however, the results are satisfactory.

Regarding the second phase, examining the recorded videos of the users revealed that younger people are more comfortable dealing with the interface. It has to be mentioned that 3 aged people tried to give vocal orders at the same time while moving their heads.

Finally, satisfaction questionnaires were given to users. The user had to evaluate, on a scale of 1 to 10 , six statements regarding the test: easy, boring, challenging, engaging, frustrating and fun. The mean and the standard deviation for each statement were calculated in order get a statistical idea about results. Table 2 depicts the questionnaire outcomes.

Table 2: Satisfaction questionnaire outcomes

\begin{tabular}{|c|c|c|c|}
\hline & Age group & Mean & Standard deviation \\
\hline \multirow{3}{*}{ Easy } & $7-15$ & 9.6 & 0.516 \\
\cline { 2 - 4 } & $16-35$ & 9 & 0.471 \\
\cline { 2 - 4 } & $36-60$ & 8.3 & 0.483 \\
\hline \multirow{3}{*}{ Boring } & $7-15$ & 0.1 & 0.316 \\
\cline { 2 - 4 } & $16-35$ & 0.2 & 0.422 \\
\cline { 2 - 4 } & $36-60$ & 0.5 & 0.527 \\
\hline \multirow{3}{*}{ Engaging } & $7-15$ & 0.1 & 0.316 \\
\cline { 2 - 4 } & $16-35$ & 0.1 & 0.316 \\
\cline { 2 - 4 } & $36-60$ & 0.2 & 0.422 \\
\cline { 2 - 4 } & $7-15$ & 9.6 & 0.699 \\
\cline { 2 - 4 } & $16-35$ & 8.9 & 0.876 \\
\hline \multirow{3}{*}{ Frustrating } & $36-60$ & 8.4 & 0.843 \\
\cline { 2 - 4 } & $7-15$ & 0 & 0 \\
\cline { 2 - 4 } & $16-35$ & 0.1 & 0.316 \\
\hline
\end{tabular}

\begin{tabular}{|c|c|c|c|}
\hline \multirow{3}{*}{ Fun } & $7-15$ & 9.9 & 0.316 \\
\cline { 2 - 4 } & $16-35$ & 8.6 & 0.843 \\
\cline { 2 - 4 } & $36-60$ & 6.6 & 0.966 \\
\hline
\end{tabular}

For the sake of simplicity, each entry is table 2 is represented as a vector (statement, age group, mean, standard deviation). The higher mean value in (fun, 7-15, 9.9, 0.316) gives a physiological indicator about the positive interactivity between the user and the design. However, the means in (easy, 7-15, 9.6, 0.516) and (engaging, 7-15, 9.6, 0.699) are quit high. The standard deviation gives an idea about the spread of the obtained results around the mean. The low standard deviation values, that exist in many places in table 2, such as in (boring, 7-15, 0.1, 0.316); indicate little variation in user's responses. However; high values indicate more variation.

\section{Conclusion and Future Work}

This paper presented the designing process of an interactive wheelchair prototype that enables quadriplegics to drive it using face gestures. The aim was to improve the quality of life for people with disability and to reduce their need for assistance from other people. Two aspects of the design were considered, the hardware and the software. The experimental results showed that young people are more comfortable using the prototype than the elderly. For the sake of convenience, the evaluation tests were conducted by healthy participants, therefore, more accurate results would require quadriplegic participants.

For a future work we are planning to design a real wheelchair that moves using the software design presented in this paper having transferred it to an android application. Moreover, vocal orders can be used as an alternative for aged people using web services. The intended design will be used in an indoor as well as an outdoor environment; therefore, a permanent connection to the internet is a must.

\section{References}

[1] S. K. Card, A. Newell and T. P. Moran, The Psychology of Human-Computer Interaction, Taylor \& Francis, 1983.

[2] F. Karray, "Human-computer interaction: Overview on state of the art," International Journal on Smart Sensing and Intelligent Systems, 2008.

[3] A. Al-Rahayfeh, M. Faezipour "Eye Tracking and Head Movement Detection: A State of-Art Survey," IEEE journal of translational engineering in health and medicine, 2013.

[4] S. U. kumar, V. M. Vinod "EOG Based Wheelchair Control for Quadriplegics," $2^{\text {nd }}$ IEEE International Conference on Innovations in Information Embedded and Communication Systems ICIIECS, 2015.

[5] T. A. Izzuddin, M. A. Ariffin, Z. H. Bohari, R. Ghazali; M. H. Jali " Movement intention detection using neural network for quadriplegic assistive machine," IEEE international Conference on Control System, Computing and Engineering (ICCDCE), 2015.

\section{Volume 7 Issue 1, January 2018 www.ijsr.net}


[6] G.Williams, Y. S. Lee, S. Ekanayake, P. N. Pathirana, L. Andriske; "Facilitating Communication and Computer Use with EEG Devices for Non-Vocal Quadriplegics" 7th International Conference on information and Automation for Sustainability (ICIAfS), 2014.

[7] A. J. Machado, J. F. Amador, M. J. Coello "Wheelchair control system for quadriplegics and ocular keyboard," The IEEE Thirty Fifth Central American and Panama Convention (CONCAPAN), 2015.

[8] J. Bray, C. F. Sturman "Bluetooth: Connect Without Cables," Prentice Hall, 2002.

[9] J. M. Hughes, "Arduino: A Technical Reference: A Handbook for Technicians, Engineers, and makers," O'Reilly, 2016.

[10] A. Kaehler, G. Bradski "Learning OpenCV 3: Computer Vision in $\mathrm{C}++$ with the OpenCV Library," O'Reilly,2016.

[11] J. Preece, H. Sharp, Y. Rogers "Interaction Design: beyond human-computer interaction," $4^{\text {th }}$ Edition, Wiley, 2015.

[12] M. F. Ruzaij, S. Neubert, N. Stoll, K. Thurow, "Design and Implementation of Low-Cost Intelligent Wheelchair Controller for Quadriplegias and Paralysis Patient," IEEE 15th International Symposium on Applied Machine Intelligence and Informatics, January 26-28, 2017.

\section{Author Profile}

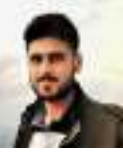

Ahmad Mahmoud is currently a fifth-year student at the Medical Engineering Faculty at Al-Andalus University, Al-Qadmous, Tartus, SYRIA.

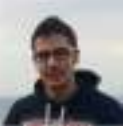

Mohammad Hamoud is currently a fifth-year student at the Medical Engineering Faculty at Al-Andalus University, Al-Qadmous, Tartus, SYRIA.

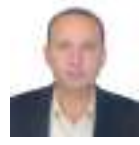

Ahmad Mahmoud Ahmad is currently a PhD lecturer in System Engineering and Computer Systems Department, Al-Baath University, Homs, SYRIA. He received the M.S. degree in Electronics and MicroElectronics from the university of Montpellier-II, France in 2005. Furthermore, he received the $\mathrm{PhD}$ degree in Networks and Telecommunication Systems from the University of Pierre and Marie Curie (Paris-6) 2010. His research interest includes Wireless Networks, Routing protocols, Netwok Security, WAN technologies and embedded systems.

Ahmad Sleiman Ahmad is currently Professor in the Medical Engineering Faculty at Al-Andalus University, Al-Qadmous, SYRIA. He received the D.E.A. degree in Industrial Control System and Human from the Valenciennes University- France and the $\mathrm{PhD}$ degree in Electrical Engineering from Ecole Centrale de LyonFrance in 1992. His research interest includes Reliability, Modelling and Simulation in electrical engineering. 